\title{
Factores asociados al desarrollo de eventos adversos en pacientes con hemodiálisis en Guerrero, México
}

\author{
Miguel Ángel Cuevas Budharta, Renata Patricia Saucedo Garcíab, José Alejandro García Larumbe ${ }^{c}$, Elsa Álvarez \\ Bolaños $^{\mathrm{d}}$, Enrique Pacheco del Cerro ${ }^{e}$, Alfonso Meneses Monroye, Mercedes Gómez del Pulgar García-Madridf, \\ Máximo A. González Juradog \\ a Enfermero del Hospital General Regional $n^{\circ} 1$ "Vicente Guerrero". Acapulco, Guerrero. México, Colaborador del \\ Instituto Español de Investigación Enfermera, Madrid. España \\ ' Unidad de Investigación Médica de Enfermedades Endocrinas del Hospital de Especialidades. CMN "Siglo XXI" \\ ' Nefrólogo de la Unidad de hemodiálisis Fresenius Medical Care, Acapulco, Guerrero. México \\ d Coordinación Delegacional de Enfermería en Atención Médica. IMSS, Guerrero. México \\ e Facultad de Enfermería, Fisioterapia y Podología de la Universidad Complutense, Madrid. España \\ ${ }^{f}$ Instituto Español de Investigación enfermera del Consejo General de Enfermería, Madrid. España \\ ${ }^{9}$ Consejo General de Enfermería, Madrid. España
}

\section{Resumen}

Objetivo: Determinar los eventos adversos más prevalentes y los factores asociados a su desarrollo en el paciente que se somete a hemodiálisis en el estado de Guerrero, México.

Material y Método: Estudio observacional, longitudinal, retrospectivo en 157 pacientes en hemodiálisis afiliados al Instituto Mexicano del Seguro Social en Guerrero, atendidos en 5 unidades privadas y 2 públicas. Se recolectaron variables socio-demográficas, mediciones de laboratorio, evolución de la enfermedad, complicaciones propias de la enfermedad y del tratamiento dialítico.

Resultados: Los eventos adversos se presentaron en el $73 \%$ de la población estudiada, la principal causa etiológica fue Diabetes mellitus tipo 2 (DM2) e hipertensión arterial. Los eventos adversos más frecuentes fueron: hipotensión $(35,5 \%)$ infección de angioacceso $(24 \%)$, cefalea $(22,3 \%)$, crisis hipertensiva $(14,5 \%)$, mareos $(9,9 \%)$, escalofríos $(9,9 \%)$, y trombosis de fístula arteriovenosa $(9,9 \%)$. Los factores que se asociaron

Correspondencia:

Miguel Ángel Cuevas-Budhart

Paseo de la Esperanza 8, 4B esc. D portal 4.28005 Madrid E-mail: angel_budhart@hotmail.com a su desarrollo fueron: pacientes mayores de 65 años, (0R=6,859IC 95\%;1,55-30,35), ser obeso, (OR=1,70, IC95\%:1,60-4,81), e hipoalbuminemia (0R=0,251, IC $95 \%: 0,160-0,593$ ).

Conclusión: Los pacientes mayores de 65 años, obesos, con hipertensión diastólica e hipoalbuminemia tienen mayor probabilidad de desarrollar eventos adversos durante el periodo de hemodiálisis.

PALABRAS CLAVE: complicaciones; diálisis renal; eventos adversos; hemodiálisis; insuficiencia renal crónica.

Factors associated with the development of adverse events in hemodialysis patients in Guerrero, Mexico

\section{Abstract}

Objective: To determine the most prevalent adverse events and the risk factors associated in the patient undergoing hemodialysis in the state of Guerrero, Mexico.

Material and Method: Observational, longitudinal, retrospective study in 157 hemodialysis patients affiliated to the Mexican Institute of Social Security in Guerrero, 
assisted in 5 private and 2 public units. Socio-demographic variables, laboratory measurements, evolution of the disease, complications of the disease and dialysis treatment were collected.

Results: Adverse events occurred in $73 \%$ of the studied population, the main etiological cause was Diabetes meIlitus type 2 (DM2) and arterial hypertension. The most frequent adverse events were: hypotension $(35.5 \%)$ angioaccess infection $(24.0 \%)$, headache $(22.3 \%)$, hypertensive crisis $(14.5 \%)$, dizziness $(9.9 \%)$, chills $(9.9 \%)$, and thrombosis of arteriovenous fistula (9.9\%). The associated risk factors: patients over $65,(0 R=6.859$, 95\% CI:1.55-30.35) being obese, (OR=1.70, 95\% CI: 1.60-4.81), and hypoalbuminemia ( $0 \mathrm{R}=0.251,95 \%$ CI:0.160-0.593).

Conclusion: Patients over 65, obese, with diastolic hypertension and hypoalbuminemia are more likely to develop adverse events during the hemodialysis.

KEYWORDS: complications; renal dialysis; adverse events; hemodialysis; chronic renal failure.

\section{Introducción}

La seguridad del paciente, componente de la calidad asistencial, ha adquirido una gran relevancia en los últimos años tanto para los pacientes y familias como para los gestores y profesionales que desean ofrecer una asistencia sanitara segura efectiva y eficiente. Así mismo, la calidad de la atención es una prioridad para las principales organizaciones internacionales de salud como la Organización Mundial de la Salud (OMS), la Unión Europea, el Consejo de Europa y para diversas autoridades sanitarias, sociedades profesionales y organizaciones de pacientes ${ }^{1-3}$.

El Ministerio de Salud y Protección Social (MPS) y la Organización Mundial de la Salud (OMS) en el 2008 definieron la seguridad del paciente como "1/a ausencia o reducción, a un nivel mínimo aceptable de riesgo de sufrir un daño innecesario de la atención sanitaria"/4. Otra definición, acuñada por el Instituto de Medicina de Estados Unidos, se refiere a: "conjunto de elementos y metodologías basadas en evidencia científicamente probada, para minimizar el riesgo de sufrir un evento adverso en el proceso de atención de salud o mitigar sus consecuencias". Como se puede observar, la seguridad está fuertemente relacionada con la calidad ${ }^{5}$. Por ello, los profesionales de la salud y en particular, el personal de enfermería, juega un papel importante en la continuidad de los cuidados y la seguridad del paciente ${ }^{6}$.

Con lo cual, la calidad de la atención en el paciente que se somete a tratamiento de sustitución renal es una prioridad, ya que, en la actualidad, la Enfermedad Renal Crónica (ERC) es un problema de salud pública mundial, debido a que se encuentra dentro de las principales causas de morbimortalidad ya que se presenta desde edades tempranas.

En México la Incidencia de la ERC se ha venido aproximando de forma abrupta y descontrolada a niveles semejantes a los encontrados en países industrializados, se calcula una prevalencia de 1.142 casos por millón de habitantes ${ }^{7}$ en población urbana y una incidencia de 377 hasta 528 casos por millón de habitantes ${ }^{8-10}$ por lo cual es considerada como una enfermedad catastrófica debido al número creciente de casos y por los altos costos de inversión, recursos de infraestructura y recursos humanos ${ }^{8}$.

Por lo cual, cada vez es mayor la necesidad de recurrir a ciertos procedimientos de sustitución renal como la hemodiálisis ${ }^{8}$. La hemodiálisis es un método de depuración extrarenal que utiliza dializadores fabricados por la industria con membranas artificiales (celulosa sintética) y que permite la supervivencia de los pacientes, sin embargo, repercute en el individuo ya que es fuente de nuevas complicaciones ${ }^{11,12}$.

Los eventos adversos (EA) asociados con la asistencia sanitaria en la población que se somete a hemodiálisis son objeto de atención en todas las organizaciones sanitarias que ofrecen este servicio ${ }^{13}$ ya que representan una causa elevada de morbimortalidad en todos los sistemas sanitarios desarrollados y que a pesar de los avances tecnológicos no ha disminuido ${ }^{14}$. La razón fundamental es la creciente complejidad en el manejo de los pacientes, en el que interactúan factores organizativos, factores personales de los profesionales y factores relacionados a la enfermedad ${ }^{2}$. Esto aumenta la demanda de hospitalizaciones generando altos costos para las instituciones de salud, al paciente y su familia, afectando el aspecto físico, psicológico y social, lo cual repercute en la calidad de vida del paciente ${ }^{15,16}$.

Existen diversos estudios que determinan los factores de riesgo relacionados a los eventos adversos por la HD. Uno de ellos son las infecciones del angioacceso en la que los factores de riesgo descritos son el tipo de acceso vascular, número de hospitalizaciones, estado nutricional, 
número de revisiones y la zona de residencia ${ }^{17-19}$. Otro tipo de EA son los eventos cardiovasculares y los factores de riesgo como la edad, y la desnutrición ${ }^{20,21}$. En otro estudio realizado en Nigeria en el que se tomó a la hipotensión intradialítica como evento adverso se asoció como factor de riesgo la nefropatía diabética ${ }^{22}$. Sin embargo, estudios sobre diversos eventos adversos y sus factores de riesgo son generalmente escasos en la literatura internacional, con lo cual, es de importancia determinar los factores de riesgo del desarrollo de EA ocurridos durante el tratamiento de hemodiálisis.

El sistema de salud en México, plantea la seguridad en el cuidado del paciente, para ello es necesario la búsqueda de conocimientos, con fines de asegurar el cuidado de las personas ${ }^{23,24}$. Por lo cual nos planteamos el objetivo siguiente: determinar los eventos adversos más prevalentes y los factores que se asocian a su desarrollo en el paciente que se somete a hemodiálisis en el estado de Guerrero, México.

\section{Material y Método}

Estudio observacional, longitudinal y retrospectivo, en pacientes sometidos a hemodiálisis en los municipios de Acapulco, Zihuatanejo, Iguala y Chilpancingo del estado de Guerrero, evaluando un total de 5 unidades privadas y 2 unidades públicas.

El muestreo fue no probabilístico por conveniencia, se incluyeron 157 pacientes que cumplieron con los criterios de selección: pacientes con más de 6 meses en tratamiento de hemodiálisis, mayores de 18 años y ambos sexos. Se analizaron las variables socio-demográficas, datos de laboratorio, evolución de la enfermedad, eventos adversos y complicaciones inherentes a la evolución de la enfermedad.

Se diseñó un instrumento el cual contenía las variables del estudio, se obtuvo el consentimiento informado por escrito de los pacientes explicándoles el objetivo del estudio y el riesgo mínimo de participar en el mismo, posteriormente se recolectó información relacionada a datos socio-demográficos a través de una entrevista cara a cara. La información relacionada con el resto de las variables, la evolución de la enfermedad y la hemodiálisis se obtuvo del expediente clínico en una búsqueda de registros clínicos hasta 6 meses de tratamiento.

La variable dependiente fue la presencia o ausencia de EA de acuerdo a la clasificación internacional para la seguridad del paciente. Dentro de las variables socio-demográficas se consideró: sexo, grupo de edad, escolaridad (categorizado en primaria, secundaria, preparatoria y licenciatura); soltero vs casado; zona urbana vs rural; ocupación; nivel socioeconómico (categorizado como nivel bajo, medio bajo, medio alto y alto).

El estudio fue presentado y aprobado por el Comité de Ética de la Delegación Guerrero del IMSS con el número de aprobación R-2016-1102-37. Se cuidó en todo momento la integridad, individualidad e intimidad de los participantes.

Los pacientes fueron informados de manera verbal sobre los objetivos e importancia de la investigación, cada rúbrica fue presentada con una hoja de consentimiento informado garantizando la confidencialidad de los resultados exclusivamente con fines estadísticos.

Análisis de los datos: Para la organización de información se diseñó una base de datos en el programa SPSS Versión 22. Se realizó un primer análisis en el cual fueron explorados los datos a través de estadística descriptiva, las variables cualitativas se describieron a través de frecuencias simples y porcentajes y las variables cuanti-

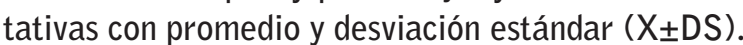

Se realizó un segundo análisis mediante estadística inferencial para determinar la relación entre las variables y la presencia de eventos adversos, se realizó un análisis estratificado en el que se calcularon las medidas de asociación tipo Odds Ratio (OR), y los intervalos de confianza al 95\%.

Por último, se realizó un tercer análisis multivariado mediante una regresión logística múltiple utilizando las variables que fueron significativas en el análisis bivariado, posteriormente se ajustó el modelo de regresión mediante el test de bondad de Hosmer Lemeshow como indicador de ajuste. Derivado del modelo se estimaron medidas de asociación mediante OR con intervalo de confianza al $95 \%$, y significancia estadística $p$ valor $<0,05$. Esto para identificar los factores que mejor explican la asociación de los factores de riesgo con los eventos adversos.

\section{Resultados}

Al comparar los grupos de pacientes entre aquellos que presentaron eventos adversos $(f=115,73 \%)$, y aquellos que no habían presentado ningún tipo de complicación, los datos son similares en cuanto a variables sociodemo- 
gráficas como género, edad, estados civil, ocupación, sin embargo, existe una variabilidad en la escolaridad. EI estado nutricional predominó un mayor porcentaje de pacientes con obesidad en el grupo de EA. En cuanto a los datos cuantitativos correspondientes a las variables clínicas existe una diferencia entre grupos, la presión arterial sistólica y diastólica se observa levemente inferior en el grupo de EA, no así para el tiempo con la enfermedad y el tiempo con HD en el que se observa superior en el grupo con presencia de EA.

Cabe señalar que los pacientes en el grupo de eventos adversos son mayores el número de pacientes que iniciaron primeramente en Diálisis peritoneal previo a su tratamiento de HD, más del $70 \%$ del grupo con EA no pudo superar 3 años en tratamiento de DP. Siendo la peritonitis recurrente la de mayor frecuencia de retiro de catéter Tenckhoff $(52,9 \%)$, (ver Tabla 1) Los principales factores etiológicos de la ERC fueron diabetes mellitus tipo 2 e hipertensión arterial.

El $77 \%$ de los pacientes presentó EA o algún tipo de complicación (ver Figura 1). Los principales eventos adversos más frecuentes fueron: hipotensión $(35,5 \%)$ infección del angioacceso (240\%), cefalea (22,3\%), crisis hipertensiva $(14,5 \%)$, mareos $(9,9 \%)$, escalofríos $(9,9 \%)$, y trombosis $(9,9 \%)$. La estenosis, aneurisma de fístula, dolor precordial y convulsiones se presentaron menor frecuencia $(0,8 \%)$. Mientras que las complicaciones inherentes a la evolución de la ERC fueron: anemia (41,5\%); hiperparatiroidismo (15,1\%); alteraciones electrolíticas $(7,5 \%)$; osteodistrofias $(11,3 \%)$; espondiloatropatías $(5,7 \%)$. La acidosis metabólica, poliglobulia y litiasis renal se presentaron con una frecuencia de 1,9\% (ver Tabla 2).

Partiendo del análisis bivariado entre las variables de interés y los pacientes con presencia de EA en los cuales se observó significancia estadística $(p<0,05)$, se incluyó en la regresión multivariable. Los factores que se asociaron a su desarrollo fueron: pacientes mayores de 65 años, (0R:6,859; IC95\%: 1,55-30,35), tener obesidad, (0R:1,70; IC95\%: 1,60-4,81) e hipoalbuminemia (OR:0,251; IC95\%: 0,160-0,593). Los datos se muestran en la Tabla 3 y Figura 2.

\section{Discusión}

El presente estudio muestra como los eventos adversos al igual que en otros países es un fenómeno que se presenta en la población de pacientes con tratamiento de sustitución renal los cuales se asocian a factores sociodemográficos, así como datos clínicos.

La presencia de Infección del angioacceso e hipotensión son los más frecuentes en la unidad de hemodiálisis. Permitiendo identificar los problemas de seguridad en las unidades de hemodiálisis en Guerrero. Estos resultados son similares a los encontrados por Mataran E, $2013^{2}$ en el que identifica los principales eventos adversos (hipotensión, infección del angioacceso, hemólisis coagulación del sistema y sin embargo, estos dos últimos no se vieron registrados en la hoja de enfermería de 6 meses de tratamiento dialítico.

Otro estudio de relevancia es el realizado por Alicia Sánchez y cols, (2012) ${ }^{25}$ en el cual tuvo una muestra de 52 pacientes encontrando el mismo espectro de complicaciones de la hemodiálisis las principales fueron; calambres $71,2 \%$ hipertensión $67,3 \%$ cefalea $55,8 \%$, hipotensión 55,85 , vómito $48,1 \%$, y disnea $34,6 \%$ y disnea $34,6 \%, 43,3 \%$, sin embargo, en comparación con el presente estudio se encuentra una discrepancia en el resultado porcentual ya que esto se debe al limitado tamaño de su muestra. Otro dato relevante es la similitud de la etiología de la ERC siendo la diabetes mellitus y la hipertensión arterial las principales causas etiológicas del deterioro de la función renal. Ambos estudios fueron realizados en población mexicana con lo cual existe una similitud innegable debido a que México ocupa el sexto lugar a nivel mundial en DM y sólo cuenta con una cuarta parte en control metabólico.

En este contexto diversos estudios determinan factores de riesgo relacionados al desarrollo de estos EA identificando como este la infección del angioacceso $0^{17-19}$ en el que determinan como factor el número de hospitalizaciones, pacientes desnutridos, número de revisiones y zona de residencia. Sin embargo, este estudio encontró como factor fuertemente relacionado pacientes mayores de 65 años, tener obesidad, e hipoalbuminemia. Los cuales tienen mayor probabilidad de sufrir un evento adverso en los pacientes que cuenta con estas variables. Cabe destacar que la obesidad puede desencadenar un conjunto de complicaciones ${ }^{26}$ por el simple hecho que conlleva ser obeso, como; intolerancia a la glucosa, aterosclerosis, dislipidemias, e hipertensión. Es importante mencionar que la obesidad es un predictor de mortalidad en el paciente renal, en este sentido los pacientes con este padecimiento que se encuentran en hemodiálisis pueden tener un mayor grado de respuesta inflamatoria con aumento de citoquinas proinflamatoria, así como una malnutrición. 
Tabla 1. Variables sociodemográficas y clínicas por grupo de pacientes.

\begin{tabular}{|c|c|c|c|c|c|c|c|c|c|c|}
\hline \multirow[b]{2}{*}{ Variable } & \multicolumn{5}{|c|}{ SIN PRESENCIA DE EA } & \multicolumn{5}{|c|}{ CON PRESENCIA DE EA } \\
\hline & $n^{0}$ & $\%$ & $\mathrm{x}$ & & DE & No & $\%$ & $\mathbf{x}$ & & DE \\
\hline $\begin{array}{l}\text { Edad } \\
\text { Género }\end{array}$ & & & 50,3 & \pm & 12,9 & & & 51,2 & \pm & 15,4 \\
\hline Masculino & 22 & 52,4 & & & & 53 & 46,1 & & & \\
\hline Femenino & 20 & 47,6 & & & & 62 & 53,9 & & & \\
\hline \multicolumn{11}{|l|}{ Estado Civil } \\
\hline Soltero & 10 & 23,8 & & & & 21 & 18,3 & & & \\
\hline Casado & 24 & 57,1 & & & & 77 & 67,0 & & & \\
\hline Divorciado & 0 & 0 & & & & 5 & 4,3 & & & \\
\hline Viudo & 3 & 7,1 & & & & 10 & 8,7 & & & \\
\hline Unión libre & 5 & 11,9 & & & & 2 & 1,7 & & & \\
\hline \multicolumn{11}{|l|}{ Escolaridad } \\
\hline Primaria & 23 & 54,8 & & & & 54 & 47,0 & & & \\
\hline Secundaria & 5 & 11,9 & & & & 25 & 21,7 & & & \\
\hline Preparatoria & 6 & 14,3 & & & & 20 & 17,4 & & & \\
\hline Licenciatura & 7 & 16,7 & & & & 15 & 13,0 & & & \\
\hline Maestría & 1 & 2,4 & & & & 1 & 0,9 & & & \\
\hline \multicolumn{11}{|l|}{ Ocupación } \\
\hline Hogar & 16 & 38,1 & & & & 48 & 41,7 & & & \\
\hline Empleado & 4 & 9,5 & & & & 12 & 10,4 & & & \\
\hline Comerciante & 7 & 16,7 & & & & 19 & 6,5 & & & \\
\hline Profesionista & 1 & 2,4 & & & & 4 & 3,5 & & & \\
\hline No trabaja & 7 & 16,7 & & & & 12 & 10,4 & & & \\
\hline Pensionista & 7 & 16,7 & & & & 20 & 17,4 & & & \\
\hline IMC & & & 24,7 & \pm & 5,1 & & & 25,2 & \pm & 4,8 \\
\hline \multicolumn{11}{|l|}{ Estado nutricio } \\
\hline Desnutrido & 3 & 7,1 & & & & 5 & 4,3 & & & \\
\hline Normal & 22 & 52,4 & & & & 55 & 47,8 & & & \\
\hline Sobrepeso & 12 & 28,6 & & & & 38 & 33,0 & & & \\
\hline Obesidad clase I & 3 & 7,1 & & & & 13 & 11,3 & & & \\
\hline Obesidad clase II & 1 & 2,4 & & & & 3 & 2,6 & & & \\
\hline Obesidad clase III & 1 & 2,4 & & & & 1 & 0,9 & & & \\
\hline \multicolumn{11}{|l|}{ Nefropatía diabética } \\
\hline $\mathrm{Si}$ & 26 & 61,9 & & & & 57 & 49,6 & & & \\
\hline No & 16 & 38,1 & & & & 58 & 50,4 & & & \\
\hline TA Sistólica & & & 151,8 & \pm & 28,0 & & & 145,1 & \pm & 26,9 \\
\hline TA Diastólica & & & 82,3 & \pm & 15,9 & & & 81,7 & \pm & 13,9 \\
\hline Tiempo con la Enfermedad Renal & & & 5,4 & \pm & 4,9 & & & 6,3 & \pm & 5,6 \\
\hline Tiempo en hemodiálisis & & & 3,4 & \pm & 3,6 & & & 3,8 & \pm & 3,1 \\
\hline \multicolumn{11}{|l|}{ Tipo de acceso venoso de HD } \\
\hline Catéter femoral & 1 & 2,4 & & & & 2 & 1,7 & & & \\
\hline Yugular & 13 & 31,0 & & & & 32 & 27,8 & & & \\
\hline Catéter subclavio & 11 & 26,1 & & & & 17 & 14,8 & & & \\
\hline Permcath & 3 & 7,1 & & & & 21 & 18,3 & & & \\
\hline Injerto & 1 & 2,4 & & & & 5 & 4,3 & & & \\
\hline Fístula & 13 & 31,0 & & & & 38 & 33,0 & & & \\
\hline \multicolumn{11}{|l|}{ Laboratorios } \\
\hline Creatinina & & & 6,581 & \pm & 3,4 & & & 7,9 & \pm & 4,0 \\
\hline Glucosa & & & 111,4 & \pm & 39,4 & & & 111,1 & \pm & 47,8 \\
\hline Albúmina & & & 3,9 & \pm & 0,7 & & & 3,7 & \pm & 0,7 \\
\hline Colesterol & & & 170,1 & \pm & 45,2 & & & 161,4 & \pm & 49,3 \\
\hline Hemoglobina & & & 10,25 & \pm & 1,9 & & & 10,3 & \pm & 2,2 \\
\hline Tiempo en DP & $\mathrm{n}=18$ & & & & & $\mathrm{n}=50$ & & & & \\
\hline$<1$ año & 3 & 16,7 & & & & 18 & 36,0 & & & \\
\hline $1-3$ años & 8 & 44,4 & & & & 19 & 38,0 & & & \\
\hline 4-6 años & 2 & 11,1 & & & & 3 & 6,0 & & & \\
\hline$>6$ años & 5 & 27,8 & & & & 10 & 20,0 & & & \\
\hline \multicolumn{11}{|l|}{ Motivo del retiro de Catéter Tenckhoff } \\
\hline Peritonitis recurrente & 10 & 55,6 & & & & 26 & 52,0 & & & \\
\hline Catéter disfuncional & 5 & 27,8 & & & & 10 & 20,0 & & & \\
\hline Infección crónica del orificio de salida & 2 & 11,1 & & & & 5 & 10,0 & & & \\
\hline Herniación & 0 & 0 & & & & 6 & 12 & & & \\
\hline$\infty 0$ tros & 1 & 5,6 & & & & 3 & 6 & & & \\
\hline
\end{tabular}

$\infty$ Retiro por trasplante, pérdida de cavidad peritoneal. Los datos cuantitativos se expresan mediante medidas de tendencia central y dispersión, los datos cualitativos se presentan con frecuencias y porcentajes. Fuente: Elaboración por el autor. 
Tabla 2. Eventos adversos y complicaciones de la ERC registradas en el expediente clínico.

\begin{tabular}{lcc}
\hline Variable & $\mathbf{f}$ & $\%$ \\
\hline Eventos adversos & & \\
Hipotensión & 43 & 35,5 \\
Infección de angioacceso & 29 & 24,0 \\
Cefalea & 27 & 22,3 \\
Crisis hipertensiva & 18 & 14,9 \\
Mareos & 12 & 9,9 \\
Escalofríos & 12 & 9,9 \\
Trombosis & 12 & 9,9 \\
Náuseas & 9 & 7,4 \\
Calambres & 9 & 7,4 \\
Caracter disfuncional & 9 & 7,4 \\
Desnutrición & 7 & 5,8 \\
O Otros & $\mathbf{5 4}$ & $\mathbf{4 4 , 6}$ \\
Complicaciones de la ERC & $\mathbf{n = 5 3}$ & \\
Anemia & 22 & 41,5 \\
Hiperparatiroidismo & 8 & 15,1 \\
Osteodistrofia & 6 & 11,3 \\
Alteraciones electrolíticas & 4 & 7,5 \\
Espondiloartropatías & 3 & 5,7 \\
- Otros & 10 & $\mathbf{1 8 , 9}$ \\
\hline
\end{tabular}

- Aneurisma de fístula. Febrícula. Arritmias. Dolor precordial. Isquemia cardiaca. Convulsiones. Estenosis. Dolor lumbar. $\bullet$. Hernia gigante por nefrectomía. Acidosis Metabólica. Gastritis crónica. Poliglobulia. Litiasis renal. Fuente: elaborado por el autor.

* Se presentaron un total de 241 eventos adversos registrados, algunos pacientes presentaron más de dos eventos adversos.

De acuerdo a los resultados de 0koye 0.C. ${ }^{22}$ en el que identificó la hipotensión intradialítica como EA, encontró como factor de riesgo la nefropatía diabética, sin embargo, en el presente estudio no se encontró ninguna asociación entre la presencia de EA y la nefropatía diabética pero, si hubo una asociación con la presencia de EA y tener hiperglucemia e hipoalbuminemia, pero al momento de realizar el modelo multivariado, solo la hipoalbuminemia resulto como factor asociado.

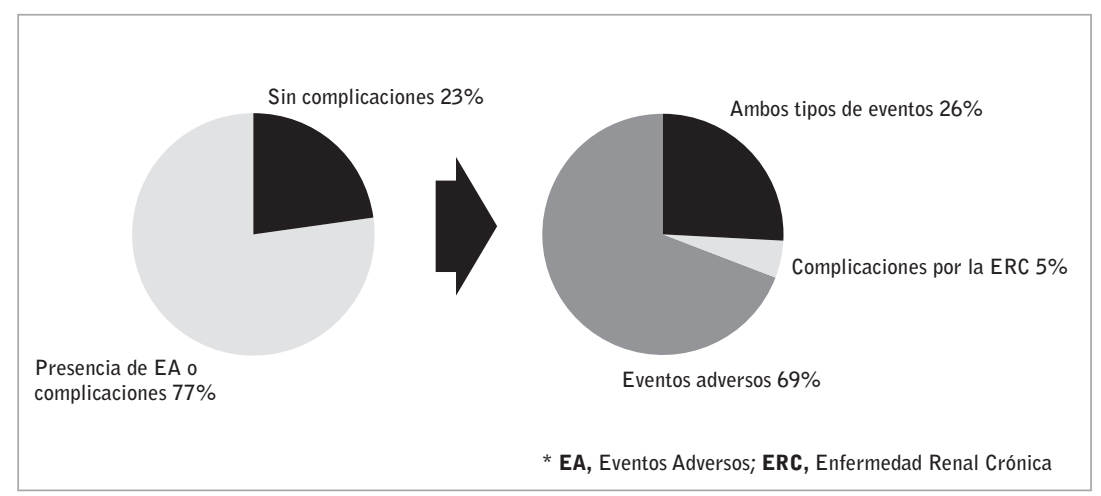

Figura 1. Porcentaje de pacientes con presencia de complicaciones y tipo de complicación.
Tabla 3. Variables socio-demográficas asociadas a la presencia de Eventos adversos en Hemodiálisis.

\begin{tabular}{|c|c|c|}
\hline Variable & \multicolumn{2}{|c|}{ Eventos adversos SI/NO } \\
\hline & $\mathbf{P}$ & Exp B (IC95\%) \\
\hline $\begin{array}{l}\text { Edad } \\
\qquad 65 \text { vs }<65\end{array}$ & 0,011 & $6,859(1,55-30,35)$ \\
\hline $\begin{array}{l}\text { Estado Civil } \\
\text { Soltero vs Casado }\end{array}$ & 0,504 & $0,734(0,297-1,817)$ \\
\hline $\begin{array}{l}\text { Educación } \\
\text { Primaria } \\
\text { Secundaria } \\
\text { Licenciatura (ref) }\end{array}$ & $\begin{array}{l}0,941 \\
0,486 \\
0,554\end{array}$ & $\begin{array}{c}0,857(0,30-3,5) \\
1,524(0,465-4.98)\end{array}$ \\
\hline $\begin{array}{l}\text { Zona } \\
\text { Rural vs Urbana }\end{array}$ & 0,423 & $1,364(0,639-2,912)$ \\
\hline $\begin{array}{l}\text { Nivel Socioeconómico } \\
\text { Bajo } \\
\text { Media bajo } \\
\text { Media alto } \\
\text { Alta (ref) }\end{array}$ & $\begin{array}{l}0,875 \\
0,387 \\
0,765 \\
0,360\end{array}$ & $\begin{array}{c}0,833(0,19-4,18) \\
2,135(0,382-11,92) \\
0,737(0,99-5,454)\end{array}$ \\
\hline $\begin{array}{l}\text { Tipo de acceso vascular } \\
\text { Catéter temporal } \\
\text { Catéter permanente } \\
\text { Fístula arterio-venosa (ref) }\end{array}$ & $\begin{array}{l}0,373 \\
0,202 \\
0,133\end{array}$ & $\begin{array}{l}0,698(0,316-1,539) \\
2,224(0,652-7,582)\end{array}$ \\
\hline Nefropatía Diabética & 0,172 & $1,654(0,803-3,404)$ \\
\hline TA Sistólica & 0,91 & $0,969(0,933-1,006)$ \\
\hline TA Diastólica & 0,045 & $(1,604(1,011-2,545)$ \\
\hline TX de inicio. DP vs HD & 0,881 & $1,082(0,385-3,038)$ \\
\hline Estado Nutricio & & \\
\hline $\begin{array}{l}\text { Desnutrición } \\
\text { Sobrepeso } \\
\text { Obeso } \\
\text { Normal (ref) }\end{array}$ & $\begin{array}{l}0,575 \\
0,344 \\
0,031 \\
0,011\end{array}$ & $\begin{array}{c}0,676(0,84-3,948) \\
1,981(0,481-8,169) \\
1,700(1,60-4,813)\end{array}$ \\
\hline
\end{tabular}

Bondad de ajuste Hosmer Lemeshow 0,627-0,993. Los pacientes pueden encontrarse en más de dos grupos ya que algunos presentaron más de dos Eventos Adversos. Fuente: Elaborado por el autor. Años en tratamiento se asoció únicamente a problemas con el flujo.

Los cuidados enfermeros prestados por el personal sanitario durante la HD son de suma importancia en el cual se debe prestar una atención de calidad, proporcionando seguridad en los procesos. Una manera de garantizar lo antes mencionado es identificar los factores que son inherentes al propio paciente, por ello, este estudio es de relevancia clínica, ya que se puede crear un proyecto educativo en los pacientes que comienzan a recibir tratamiento de hemodiálisis y que esto permita una mejor evolución de la enfermedad. 


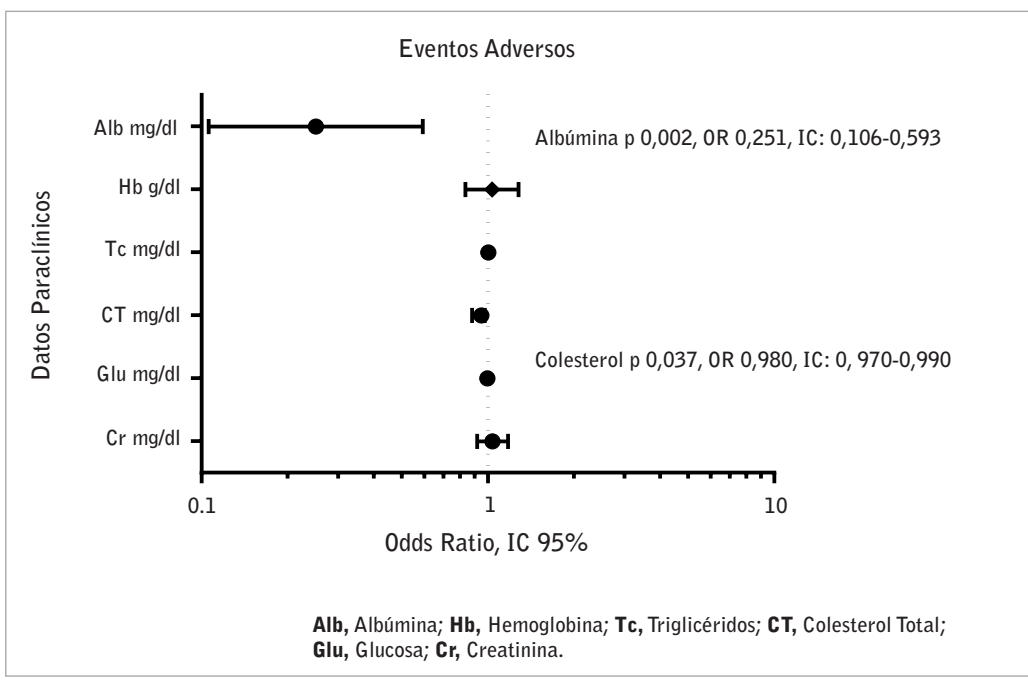

Figura 2. Parámetros de laboratorio asociados a los eventos adversos.

Una limitación del estudio es que se realizó de manera retrospectiva y transversal por lo cual no se pueden realizar pruebas estadísticas para determinar los factores de riesgo por ello, se proponen realizar estudios de cohorte prospectivos en los que se tomen más variables clínicas y en las que se pueda realizar test estadísticos de supervivencia. Cabe señalar que otros estudios mencionan como los niveles de parathormona y el fósforo sérico se convierte en un alto riesgo cardiovascular, lo que amerita un mayor estudio a profundidad para determinar otros factores bioquímicos relacionados a esta complicación.

A partir de los resultados de nuestro estudio podemos observar, que los pacientes con Enfermedad Renal Crónica que se encuentran en tratamiento de hemodiálisis presentan un alto índice de EA durante el periodo de su tratamiento. Los eventos adversos más frecuentes son hipotensión, infección de angioacceso, cefalea y crisis hipertensiva.

Los factores sociodemográficos que más se asocia al desarrollo de eventos adversos son, Pacientes mayores de 65 años. Las variables médicas de mayor asociación a la presencia de EA son: presión arterial Diastólica, obesidad e hipoalbuminemia. Respecto a la atención sanitaria solo se asoció fuertemente el tipo de Acceso vascular y el paciente que inicia primeramente en diálisis peritoneal, sin embargo, en el modelo multivariado no se encontró asociación con los EA.

Los centros de Hemodiálisis deben contar con las herramientas necesarias de evaluación y atención al paciente para identificar los factores de riesgo. La coordinación y la gestión clínica de procesos de los pacientes sometidos a hemodiálisis debe ser una estrategia multidisciplinaria para garantizar la seguridad del paciente.

\section{Conflictos de interés}

Los autores declaran no tener ningún conflicto de intereses en lo que corresponde a la investigación, la autoría o la publicación de este artículo.

\section{Financiación}

Esta investigación no recibió ninguna subvención específica de las agencias de financiamiento en los sectores público, comercial o sin fines de lucro. Obra de arte electrónica.

Recibido: 7 noviembre 2018

Revisado: 5 febrero 2019

Modificado: 6 febrero 2019

Aceptado: 10 febrero 2019

\section{Bibliografía}

1. Agencia de Calidad del Sistema Nacional de Salud. Análisis de la cultura sobre seguridad del paciente en el ámbito hospitalario del Sistema Nacional de Salud.. Madrid: Ministerio de Sanidad y Política Social [Internet]. 2009 [Consultado 10 0ct 2018]. Disponible en: http://www.mscbs.gob.es/organizacion/sns/planCalidadSNS/docs/Analisis_cultura SP_ambito_hospitalario.pdf

2. Matarán $E$, Aguilar $R$, Muñoz M. Incidencia y tipo de efectos adversos durante el procedimiento de hemodiálisis. Enferm Nefrol. 2013;16(1):36-40.

3. Sanidad M de. Estrategia de Seguridad del Paciente del Sistema Nacional de Salud 2015-2020. Minist Sanidad, Serv Soc e Igual [Internet]. 2015 [consultado 12 0ct 2018];1-128. Disponible en: https://www. seguridaddelpaciente.es/resources/documentos/2015/ Estrategia Seguridad del Paciente 2015-2020.pdf 
4. Conceptual Framework for the International Classification for Patient Safety Version 1.1 Final Technical Report. World Health Organization \& WHO Patient Safety [Internet]. 2009 [consultado 10 0ct 2018]. Disponible en: https://www.who.int/patientsafety/taxonomy/icps_full_report.pdf

5. Górriz JL, Sancho A, Pallardó LM, Amoedo ML, Martín M, Sanz P, et al. Significado pronóstico de la diálisis programada en pacientes que inician tratamiento sustitutivo renal. Un estudio multicéntrico español. Nefrologia. 2002;22(1):49-59.

6. Torres Santiago LM, Zárate Grajales MRA, Matus Miranda MR. Calidad de los registros clínicos de enfermería: Elaboración de un instrumento para su evaluación. Enfermería Univ [Internet]. 2011 [consultado 10 Oct 2018];8(1):17-25. Disponible en: http://www.revistas.unam.mx/index.php/reu/article/ view/25467.

7. Méndez-Durán A, Francisco Méndez-Bueno J, Tapia-Yáñez T, Montes AM, Aguilar-Sánchez L. Epidemiología de la insuficiencia renal crónica en México. Diálisis y Traspl. 2010;31(1):7-11.

8. Méndez-Durán A, Pérez-Aguilar $G$, Ayala-Ayala $F$, Ruiz-Rosas RA, González-Izquierdo J de J, Dávila-Torres J. Panorama epidemiológico de la insuficiencia renal crónica en el segundo nivel de atención del Instituto Mexicano del Seguro Social. Diálisis y Traspl. 2014;35(4):148-56.

9. Amato D, Alvarez-Aguilar C, Castañeda-Limones $R$, Rodriguez E, Avila-Diaz M, Arreola F, et al. Prevalence of chronic kidney disease in an urban Mexican population. Kidney Int Suppl. 2005;(97):S11-7.

10. United States Renal Data System. 2016 USRDS annual data report: Epidemiology of kidney disease in the United States. National Institutes of Health, National Institute of Diabetes and Digestive and Kidney Diseases [Internet]. 2016 [consultado 10 Oct 2018]. Disponible en: https://www.usrds. org/2016/view/Default.aspx

11. Ward RA. Do Clinical Outcomes in Chronic Hemodialysis Depend on the Choice of a Dialyzer? Semin Dial. 2011;24(1):65-71.
12. Mezzano A. S, Aros E. C. Enfermedad renal crónica: clasificación, mecanismos de progresión y estrategias de renoprotección. Rev Méd Chile 2005;133(3):338-48.

13. Homsted L. Institute of Medicine report: to err is human: building a safer health care system. The $\mathrm{Na}$ tional Academies Press; [Internet] 2000 [consultado 10 0ct 2018]. Disponible en: https://www.nap. edu/read/9728/chapter/1.

14. Herzog C a, Ma JZ, Collins a J. Poor long-term survival after acute myocardial infarction among patients on long-term dialysis. N Engl $\mathrm{J}$ Med. 1998:339:799-805.

15. Gorostidi M, Santamaría $R$, Alcázar R, Fernández-Fresnedo G, Galcerán JM, Goicoechea M, et al. Documento de la sociedad española de nefrología sobre las guías KDIGO para la evaluación y el tratamiento de la enfermedad renal crónica. Nefrologia. 2014;34(3):273-424.

16. Cuevas-Budhart MA, Saucedo García RP, Romero Quechol G, García Larumbe JA, Hernández Paz y Puente $A$. Relación entre las complicaciones y la calidad de vida del paciente en hemodiálisis. Enferm Nefrol 2017;20(2):112-9.

17. Bonomo RA, Rice D, Whalen C, Linn D, Eckstein E, Shlaes DM. Risk factors associated with permanent access-site infections in chronic hemodialysis patients. Infect Control Hosp Epidemiol. 1997;18(11):757-61.

18. Dalrymple LS, Mu Y, Nguyen DV, Romano PS, Chertow GM, Grimes B, et al. Risk factors for infection-related hospitalization in in-center hemodialysis. Clin J Am Soc Nephrol. 2015;10(12):2170-80.

19. Quori A, Baamonde-Laborda E, García-Cantón C, Lago-Alonso M M, Toledo-González A, Monzón-Jiménez $E$, et al. Vigilancia de infecciones y otros eventos adversos en pacientes en diálisis en el área sur de Gran Canaria. Nefrologia. 2011;31(4):457-63.

20. Collado $S$, Coll E, Deulofeu $R$, Guerrero $L$, Pons M, Cruzado JM, et al. Prevalencia de enfermedad cardiovascular en la uremia y relevancia de los factores de riesgo cardiovascular. Nefrologia. 2010;30(3):342-8. 
21. Shah DS, Polkinghorne $K R$, Pellicano $R$, Kerr PG. Are traditional risk factors valid for assessing cardiovascular risk in end-stage renal failure patients? Nephrology (Carlton). 2008;13(8):667-71.

22. Okoye OC, Slater $H E$, Rajora N. Prevalence and risk factors of intra-dialytic hypotension: A 5 year retrospective report from a single Nigerian centre. Pan Afr Med J. 2017;28.

23. Araujo Ruiz JA, Arencibia Jorge R, Gutiérrez Calzado $C$. Ensayos clínicos cubanos publicados en revistas de impacto internacional: estudio bibliométrico del período 1991-2001. Rev. Esp. Doc. Cient [Internet]. 2002 [consultado 10 0ct 2018];25(3):25466. Disponible en: http://redc.revistas.csic.es/index. php/redc/article/view/103/168dDoc.csic.es:8080stas.csic.es/index.php/redc/article/view/103/168.

24. Fajardo-Dolci G, Rodríguez-Suárez J, Campos-Castolo $\mathrm{M}$, Carrillo-Jaimes A, Zavala-Suárez E, Aguirre-Gas $\mathrm{H}$. Lineaminetos generales para el cuidado de la seguridad del paciente. Rev CONAMED [Internet]. 2008 [consultado 12 0ct 2018];13(3):3846. Disponible en: http://www.conamed.gob.mx/publicaciones/pdf/REVISTA_JUL-SEP_2008.pdf
25. Sánchez-García A, Zavala-Méndez MC, Pérez-Pérez A. Hemodiálisis: proceso no exento de complicaciones. Rev Enferm Inst Mex Seguro Soc. 2012;20(3):131-7.

26. Cases A. Otros factores de riesgo cardiovascular y renal. Hipertrofia del ventrículo izquierdo. fibrilación auricular. Tabaquismo. Obesidad. factores emergentes de riesgo cardiovascular: Homocisteína. Proteína c reactiva. Fibrinógeno. Nefrologia. 2004;24(Supl6):S62-72.

Este artículo se distribuye bajo una Licencia Creative Commons Atribución-NoComercial 4.0 Internacional. https://creativecommons.org/licenses/by-nc/4.0/

Open Access (C) () () 